\title{
Transient Free Convective MHD Flow Past an Exponentially Accelerated Vertical Porous Plate with Variable Temperature through a Porous Medium
}

\author{
Ashish Paul \\ Department of Mathematics, Cotton College State University, Guwahati 781001, India \\ Correspondence should be addressed to Ashish Paul; ashpaul85@gmail.com
}

Received 30 June 2016; Accepted 30 November 2016; Published 17 January 2017

Academic Editor: Alberto Cardona

Copyright (C) 2017 Ashish Paul. This is an open access article distributed under the Creative Commons Attribution License, which permits unrestricted use, distribution, and reproduction in any medium, provided the original work is properly cited.

\begin{abstract}
This paper is concerned with analytical solution of one-dimensional unsteady laminar boundary layer MHD flow of a viscous incompressible fluid past an exponentially accelerated infinite vertical plate in presence of transverse magnetic field. The vertical plate and the medium of flow are considered to be porous. The fluid is assumed to be optically thin and the magnetic Reynolds number is considered small enough to neglect the induced hydromagnetic effects. The governing boundary layer equations are first converted to dimensionless form and then solved by Laplace transform technique. Numerical values of transient velocity, temperature, skin friction, and Nusselt number are illustrated and are presented in graphs for various sets of physical parametric values, namely, Grashof number, accelerating parameter, suction parameter, permeability parameter, radiation parameter, magnetic parameter, and time. It is found that the velocity decreases with increases of the suction parameter for both cases of cooling and heating of the porous plate whereas skin friction increases with increase of suction parameter.
\end{abstract}

\section{Introduction}

The study of unsteady natural convective flow of viscous incompressible fluid past vertical bodies has wide engineering and technological applications. When free convection flows occurs at high temperature, the effects of radiation are vital important. Thermal radiation is key to many fundamental phenomena surrounding us, from solar radiation to fire incandescent lamp, and has played a major role in combustion and furnace design, design of fins, nuclear power plants, cooling of towers, gas turbines and various propulsion device for aircraft, energy utilization, temperature measurements, remote sensing for astronomy, and space exploration.

Magnetohydrodynamic (MHD) flow and heat and mass transfer processes occur in many industrial applications such as the geothermal system, aerodynamic processes, chemical catalytic reactors and processes, electromagnetic pumps, and MHD power generators. Many studies have been carried out to investigate the magnetohydrodynamic transient free convective flow. Gupta [1] first studied transient free convection of an electrically conducting fluid from a vertical plate in the presence of magnetic field. Free convection effects on flow past an exponentially accelerated vertical plate was studied by Singh and Kumar [2]. Jha et al. [3] analyzed mass transfer effects on exponentially accelerated infinite vertical plate with constant heat flux and uniform mass diffusion. Muthucumaraswamy et al. [4] studied the flow past an exponentially accelerated infinite vertical plate in the presence of variable surface temperature. Again Muthucumaraswamy et al. [5] analysed the combined effects of heat and mass transfer on exponentially accelerated vertical plate in presence of uniform magnetic field. Rajesh et al. [6] presented the analytic investigation of radiation and mass transfer effects on MHD free convection flow past an exponentially accelerated vertical plate in the presence of variable mass diffusion. Rajesh and Chamkha [7] presented numerical solution to study the unsteady convective flow past an exponentially accelerated infinite vertical porous plate with Newtonian heating and viscous dissipation by Galerkin finite element method. They have found that the skin friction coefficient increases with increases in either of the Eckert number. Recently, Srinivasa et al. [8] presented 
both numerical and analytical solutions to study the effect of chemical reaction on unsteady, incompressible, viscous flow past an exponentially accelerated vertical plate with heat absorption and variable temperature in a magnetic field.

In recent years convective heat transfer in porous media has attracted considerable attention owing to its wide industrial and technological applications such as geothermal energy recovery, oil extraction, fibre and granular insulation, electronic system cooling, and porous material regenerative heat exchangers. Gupta et al. [9] have studied free convection on flow past an accelerated vertical plate in the presence of viscous dissipative heat using perturbation method. Kafousias and Raptis [10] extended their (Gupta et al. [9]) work by including mass transfer effects subjected to variable suction or injection. Transient free convection flow past a plate embedded in a porous medium pioneered by Ping and Pop [11]. Jha [12] presented analytic investigation of uniform transverse magnetic field effect on the free-convection and mass-transform flow of an electrically conducting fluid past an infinite vertical plate for uniformly accelerated motion of the plate through a porous medium. Magyari et al. [13] have presented an analytical solution for unsteady free convection in porous media. Chaudhary and Jain [14-16] and Chaudhary et al. [17] analyzed MHD transient free convection effects on flow past a moving vertical plate embedded in porous medium under different physical situations by employing Laplace transform technique. Rajesh [18] presented an analytic investigation of MHD free convection flow past an accelerated vertical porous plate with variable temperature through a porous medium. Again Rajesh et al. [19] studied the transient free convection MHD flow and heat transfer of nanofluid past an impulsively started vertical porous plate in the presence of viscous dissipation. Recently, Ibrahim [20] studied the effects of thermal radiation, viscous dissipation, and chemical reaction on unsteady two-dimensional boundary layer MHD flow over a nonisothermal stretching sheet embedded in porous medium.

However, to the best of authors' knowledge, MHD free convective flow past an exponentially accelerated vertical porous plate with variable temperature through a porous medium was never considered in the literature. The objective of this paper is to study magnetohydrodynamic transient heat transfer flow past an exponentially accelerated infinite vertical porous plate with variable temperature and the plate is embedded in a porous medium. The exact solutions of the dimensionless unsteady linear governing equations are obtained by Laplace transform technique.

\section{Mathematical Analysis}

An unsteady one-dimensional laminar free convective flow of a viscous incompressible fluid past an infinite vertical porous plate through a porous medium with variable temperature is considered. The $x$-axis is being taken vertically upwards along the vertical plate and $y$-axis is taken to be normal to the plate. The physical model and coordinate system of the flow problem is shown in Figure 1. Initially, it is assumed that the plate and fluid are at the same temperature $T_{\infty}^{\prime}$ in the stationary condition. At $t^{\prime} \geq 0$, the plate is exponentially

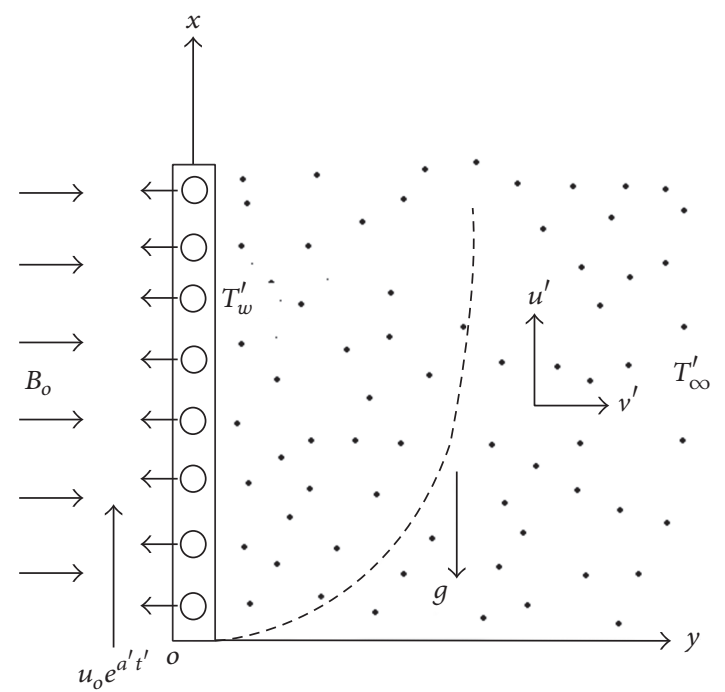

Figure 1: Physical model and coordinate system.

accelerated with a velocity $u^{\prime}=u_{0}^{\prime} \exp \left(a^{\prime} t^{\prime}\right)$ in its own plane and the plate temperature is raised linearly with time $t$. A uniform magnetic field is applied in the direction perpendicular to the plate. The fluid is assumed to be slightly conducting, so that the magnetic Reynolds number is much less than unity and hence the induced magnetic field is negligible in comparison with the applied magnetic field. The fluid considered here is a gray, absorbing/emitting radiation but a nonscattering medium. The viscous dissipation is also assumed to be negligible in the energy equation as the motion is due to free convection only. It is also assumed that all the fluid properties are constant except for the density in the buoyancy term, which is given by the usual Boussinesq's approximation. Under these assumptions the governing boundary layer equations are

$$
\begin{aligned}
\frac{\partial u^{\prime}}{\partial t^{\prime}}+v^{\prime} \frac{\partial u^{\prime}}{\partial y^{\prime}}= & \nu \frac{\partial^{2} u^{\prime}}{\partial y^{\prime 2}}+g \beta\left(T^{\prime}-T_{\infty}^{\prime}\right) \\
& -\frac{\sigma B_{0}^{2}}{\rho} u^{\prime}-v \frac{u^{\prime}}{k^{\prime}} \\
\rho C_{p}\left(\frac{\partial T^{\prime}}{\partial t^{\prime}}+v^{\prime} \frac{\partial T^{\prime}}{\partial y^{\prime}}\right)= & \kappa \frac{\partial^{2} T^{\prime}}{\partial y^{\prime 2}}-\frac{\partial q_{r}}{\partial y^{\prime}}
\end{aligned}
$$

with the following initial and boundary conditions:

$$
\begin{array}{rlr}
t^{\prime} \leq 0: & u^{\prime}=0, & \\
T^{\prime}=T_{\infty}^{\prime} & \forall y^{\prime}, \\
t^{\prime}>0: & u^{\prime}=u_{0} \exp \left(a^{\prime} t^{\prime}\right), & \\
T^{\prime}=T_{\infty}^{\prime}\left(T^{\prime}{ }_{w}-T_{\infty}^{\prime}\right) A t^{\prime}, & \\
u^{\prime} \longrightarrow 0, & \text { at } y^{\prime}=0, \\
& T^{\prime} \longrightarrow T_{\infty}^{\prime},
\end{array}
$$

as $y \longrightarrow \infty$,

where $A=u_{0}^{2} / \nu$. 
The local radiant for the case of an optically thin gray gas is expressed by

$$
\frac{\partial q_{r}}{\partial y^{\prime}}=-4 a^{*} \sigma\left(T_{\infty}^{\prime 4}-T^{\prime 4}\right)
$$

We assume that the temperature differences within the flow are sufficiently small such that $T^{\prime 4}$ may be expressed as a linear function of the temperature. This is accomplished by expanding $T^{\prime 4}$ in a Taylor series about $T_{\infty}^{\prime}$ and neglecting higher-order terms; thus

$$
T^{\prime 4}=-4 T_{\infty}^{\prime 3} T^{\prime}-3 T_{\infty}^{\prime 4} .
$$

By using (4) and (5), (2) gives

$$
\rho C_{p} \frac{\partial T^{\prime}}{\partial t^{\prime}}=\kappa \frac{\partial^{2} T^{\prime}}{\partial y^{\prime 2}}-16 a^{*} \sigma T_{\infty}^{\prime 3}\left(T_{\infty}^{\prime}-T^{\prime}\right) .
$$

In order to write the governing equations, initial and boundary conditions in dimensionless form, the following nondimensional quantities are introduced:

$$
\begin{aligned}
& U=\frac{u^{\prime}}{u_{0}}, \\
& Y=\frac{y^{\prime} u_{0}}{v}, \\
& t=\frac{t^{\prime} u_{0}}{v} \\
& T=\frac{T^{\prime}-T_{\infty}^{\prime}}{T_{w}^{\prime}-T_{\infty}^{\prime}}, \\
& \operatorname{Pr}=\frac{\mu C_{p}}{\kappa}, \\
& \mathrm{Gr}=g \beta v \frac{T_{w}^{\prime}-T_{\infty}^{\prime}}{u_{0}^{3}}, \\
& M=\frac{\sigma B_{0}^{2} r_{0}^{2}}{v \rho}, \\
& k=\frac{k^{\prime} u_{0}^{2}}{v^{2}}, \\
& R=\frac{16 a^{*} v^{2} \sigma T_{\infty}^{\prime 3}}{\kappa u_{0}^{2}}, \\
& a=\frac{a^{\prime} v}{u_{0}^{2}}, \\
& \gamma=-\frac{v^{\prime}}{u_{0}} .
\end{aligned}
$$

In view of (7), the governing equations (1) and (6) reduce to the following nondimensional form:

$$
\begin{aligned}
& \frac{\partial U}{\partial t}-\gamma \frac{\partial U}{\partial Y}=\frac{\partial^{2} U}{\partial Y^{2}}+\operatorname{Gr} T-M U-\frac{U}{k} \\
& \frac{\partial T}{\partial t}-\gamma \frac{\partial T}{\partial Y}=\frac{1}{\operatorname{Pr}} \frac{\partial^{2} T}{\partial Y^{2}}-\frac{R}{\operatorname{Pr}} T
\end{aligned}
$$

with following initial and boundary conditions:

$$
\begin{array}{r}
t \leq 0: U=0, \\
T=0,
\end{array}
$$

$\forall Y$,

$$
\begin{aligned}
t>0: U & =\exp (a t), \\
T & =t,
\end{aligned}
$$

$$
\text { at } Y=0 \text {, }
$$

$$
U \longrightarrow 0
$$$$
T \longrightarrow 0 \text {, }
$$

$$
\text { as } Y \longrightarrow \infty \text {. }
$$

All physical variables and parameters are mentioned in nomenclature.

\section{Solution Technique}

To solve the unsteady equations (8) and (9) subject to initial and boundary conditions (10), we apply Laplace transform technique for the case unit Parndtl number.

Laplace transforms of (8) and (9) give rise to

$$
\begin{aligned}
\frac{d^{2} \bar{U}}{d Y^{2}}+\gamma \frac{d \bar{U}}{d Y}- & \left(p+M+\frac{1}{k}\right) \bar{U}+\mathrm{Gr} \bar{T}=0, \\
\frac{d^{2} \bar{T}}{d Y^{2}}+\gamma \frac{d \bar{T}}{d Y}-(p+R) \bar{T} & =0,
\end{aligned}
$$

where $p$ is the Laplace transformation parameter and $\bar{U} \& \bar{T}$ are the Laplace transform of $U \& T$, respectively.

Solutions of (11) and (12) subject to initial and boundary conditions (10) give

$$
\begin{aligned}
\bar{U}= & \frac{1}{p-a} e^{-Y((\gamma / 2)+\sqrt{p+d})}+\frac{\mathrm{Gr}}{L} \frac{e^{-Y((\gamma / 2)+\sqrt{p+d})}}{p^{2}} \\
& -\frac{\mathrm{Gr}}{L} \frac{e^{-Y((\gamma / 2)+\sqrt{p+S})}}{p^{2}}, \\
\bar{T}= & \frac{1}{p^{2}} e^{-Y((\gamma / 2)+\sqrt{p+S})} .
\end{aligned}
$$


Inverse Laplace transforms of (13) and (14), respectively, give the velocity and temperature profiles as

$$
\begin{aligned}
U & =\frac{e^{a t}}{2}\left\{e^{-Y((\gamma / 2)+\sqrt{a+d})} \operatorname{erfc}\left(\frac{Y}{2 \sqrt{t}}-\sqrt{(a+d) t}\right)\right. \\
& \left.+e^{-Y((\gamma / 2)-\sqrt{a+d})} \operatorname{erfc}\left(\frac{Y}{2 \sqrt{t}}+\sqrt{(a+d) t}\right)\right\} \\
& +\frac{\operatorname{Gr}}{L}\left(\frac{t}{2}-\frac{Y}{4 \sqrt{d}}\right) \exp \left(-Y\left(\frac{\gamma}{2}+\sqrt{d}\right)\right) \\
& \cdot \operatorname{erfc}\left(\frac{Y}{2 \sqrt{t}}-\sqrt{d t}\right)+\frac{\operatorname{Gr}}{L}\left(\frac{t}{2}+\frac{Y}{4 \sqrt{d}}\right) \\
& \cdot \exp \left(-Y\left(\frac{\gamma}{2}-\sqrt{d}\right)\right) \operatorname{erfc}\left(\frac{Y}{2 \sqrt{t}}+\sqrt{d t}\right) \\
& -\frac{\operatorname{Gr}}{L}\left(\frac{t}{2}-\frac{Y}{4 \sqrt{S}}\right) \exp \left(-Y\left(\frac{\gamma}{2}+\sqrt{S}\right)\right) \operatorname{erfc}\left(\frac{Y}{2 \sqrt{t}}\right. \\
& -\sqrt{S t})-\frac{\operatorname{Gr}}{L}\left(\frac{t}{2}+\frac{Y}{4 \sqrt{S}}\right) \exp \left(-Y\left(\frac{\gamma}{2}-\sqrt{S}\right)\right) \\
& -\operatorname{erfc}\left(\frac{Y}{2 \sqrt{t}}+\sqrt{S t}\right) \cdot \\
& \cdot \operatorname{erfc}\left(\frac{Y}{2 \sqrt{t}}+\sqrt{S t}\right) \\
& \left(\frac{t}{2}-\frac{Y}{4 \sqrt{S}}\right) \exp \left(-Y\left(\frac{\gamma}{2}+\sqrt{S}\right)\right) \operatorname{erfc}\left(\frac{Y}{2 \sqrt{t}}\right. \\
& \\
&
\end{aligned}
$$

Knowing the velocity and temperature field, it is very interesting to study the skin friction and Nusselt number. In nondimensional form the skin friction and Nusselt number are defined, respectively, as follows:

$$
\begin{gathered}
\tau=-\left.\frac{\partial U}{\partial Y}\right|_{Y=0}, \\
\mathrm{Nu}=-\left.\frac{\partial T}{\partial Y}\right|_{Y=0} .
\end{gathered}
$$

3.1. Skin Friction. Expression of the skin-friction $\tau$ is obtained from (15) as

$$
\begin{aligned}
\tau & =\frac{e^{-d t}}{\sqrt{\pi t}}+\frac{e^{a t}}{2}\left\{\left(\frac{\gamma}{2}+\sqrt{a+d}\right) \operatorname{erfc}(-\sqrt{t(a+d)})\right. \\
& \left.+\left(\frac{\gamma}{2}-\sqrt{a+d}\right) \operatorname{erfc}(\sqrt{t(a+d)})\right\} \\
& +\frac{\operatorname{Gr} t}{2 L}\left\{\left(\frac{\gamma}{2}+\sqrt{d}\right) \operatorname{erfc}(-\sqrt{d t})\right. \\
& \left.+\left(\frac{\gamma}{2}-\sqrt{d}\right) \operatorname{erfc}(\sqrt{d t})\right\} \\
& +\frac{\operatorname{Gr} t}{2 L}\left\{\left(\frac{\gamma}{2}+\sqrt{S}\right) \operatorname{erfc}(-\sqrt{S t})\right. \\
& \left.+\left(\frac{\gamma}{2}-\sqrt{S}\right) \operatorname{erfc}(\sqrt{S t})\right\}+\frac{\operatorname{Gr}}{4 L \sqrt{d}}\{\operatorname{erfc}(-\sqrt{d t}) \\
& -\operatorname{erfc}(\sqrt{d t})\}-\frac{\operatorname{Gr}}{4 L \sqrt{S}}\{\operatorname{erfc}(-\sqrt{S t}) \\
& -\operatorname{erfc}(\sqrt{S t})\}+\frac{\operatorname{Gr} \sqrt{t}}{L \sqrt{\pi}}\{\exp (-d t)-\exp (-S t)\} .
\end{aligned}
$$

3.2. Nusselt Number. Expression of Nusselt number $\mathrm{Nu}$ is obtained from (16) as

$$
\begin{aligned}
\mathrm{Nu} & =\frac{t}{2}\left\{\left(\frac{\gamma}{2}+\sqrt{S}\right) \operatorname{erfc}(-\sqrt{S t})\right. \\
& \left.+\left(\frac{\gamma}{2}-\sqrt{S}\right) \operatorname{erfc}(\sqrt{S t})\right\}-\frac{1}{4 \sqrt{S}}\{\operatorname{erfc}(-\sqrt{S t}) \\
& -\operatorname{erfc}(\sqrt{S t})\}+\sqrt{\frac{t}{\pi}} \exp (-S t),
\end{aligned}
$$

where $d=\gamma^{2} / 4+M+1 / k, S=\gamma^{2} / 4+R, L=R-M-1 / k$.

\section{Results and Discussion}

In order to get an insight into the physical solution of the problem, the numerical computations of velocity profile, temperature profile, skin friction, and Nusselt number are obtained for different values of magnetic field parameter $M$, Grashof numbers Gr, accelerating parameter $a$, suction parameter $\gamma$, permeability parameter $k$, radiation parameter $R$, and time $t$ and presented graphically in Figures 2-14.

The transient velocity profiles for different values of Grashof number at $a=0.6, M=1.2, k=0.3, R=2$, $t=0.6$, and $\gamma=0.4$ are shown in Figure 2. The Grashof number signifies the relative effect of the buoyancy force to the hydrodynamic viscous force. The positive values of $\mathrm{Gr}$ correspond to cooling of the plate and the negative values of $\mathrm{Gr}$ correspond to heating of the plate by free convection. As expected, it is found that an increase in the Grashof number leads to increase the velocity due to enhancement in the buoyancy force. 


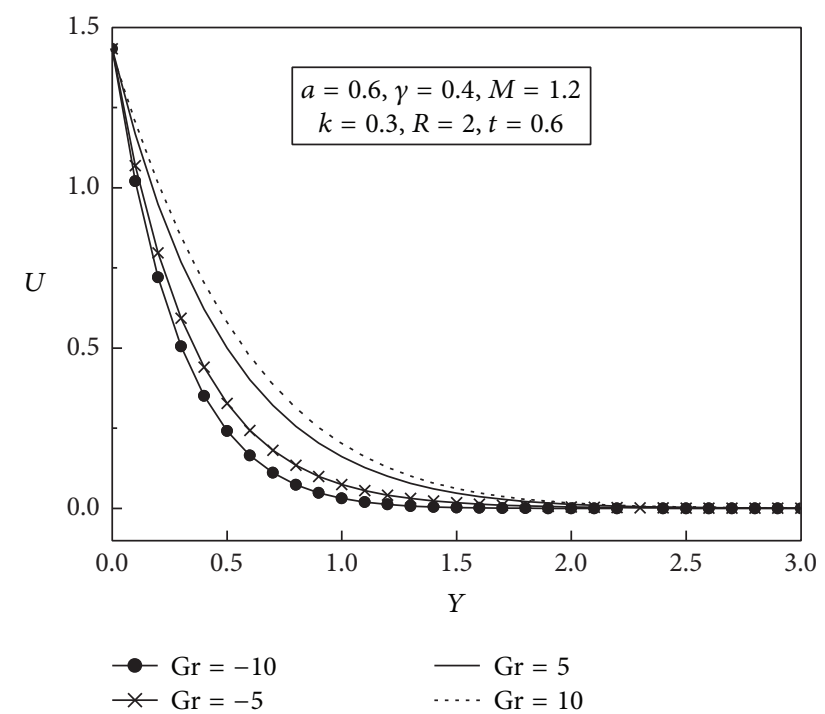

FigURE 2: Effect of $\mathrm{Gr}$ on velocity profiles at $a=0.6, M=1.2, k=$ $0.3, R=2, t=0.6$, and $\gamma=0.4$.

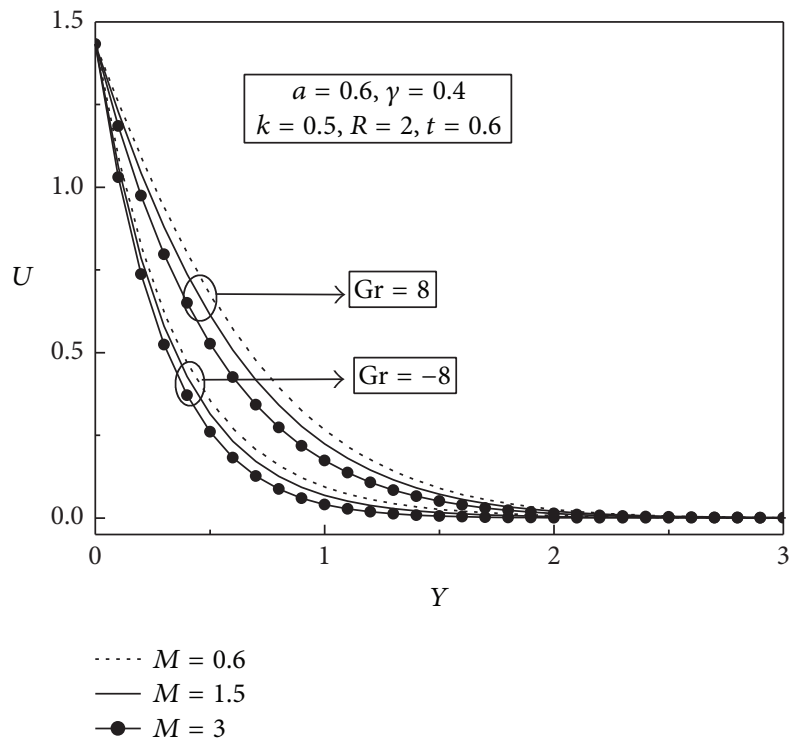

Figure 3: Effect of $M$ on velocity profiles at $\mathrm{Gr}=(-8 \& 8), a=0.6$, $k=0.5, R=2, t=0.6$, and $\gamma=0.4$.

The transient velocity profiles for different values of Magnetic parameter $M$ at $\mathrm{Gr}=-8 \& 8, a=0.6, k=0.5$, $R=2, t=0.6$, and $\gamma=0.4$ are shown in Figure 3. It is observed from the figure that an increase in magnetic field leads to decrease in the velocity field for both the cases of cooling and heating of the porous plate. It is because that the application of transverse magnetic field will result a resistive type force (Lorentz force) similar to drag force which tends to resist the fluid flow and thus reducing its velocity.

The effects of suction parameter $\gamma$ on velocity profiles at $\mathrm{Gr}=-8 \& 8, a=0.6, k=0.5, t=0.6, R=2$, and $M=1.5$ are

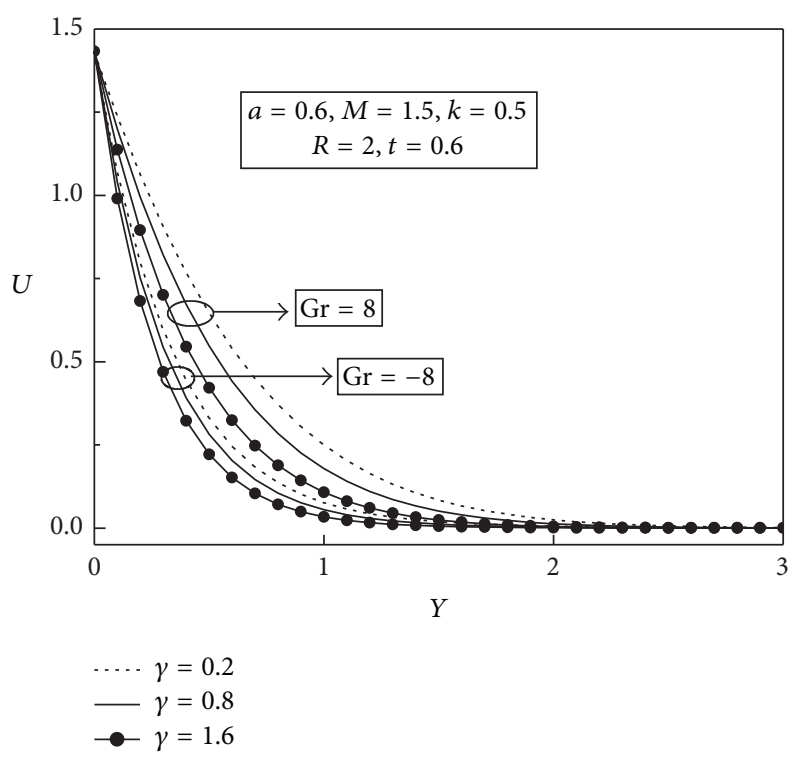

FIGURE 4: Effect of $\gamma$ on velocity profiles at $\mathrm{Gr}=(-8 \& 8), a=0.6$, $k=0.5, t=0.6, R=2$, and $M=1.5$.



FIgURE 5: Effects of $k$ and $t$ on velocity profiles at $\mathrm{Gr}=5, a=0.6$, $R=2, M=1.5$, and $\gamma=0.4$.

shown in Figure 4. It is found here that velocity decreases with increases of the suction parameter $\gamma$ for both cases of cooling and heating of the porous plate. The effects of permeability parameter $k$ and time $t$ on velocity profiles at $\mathrm{Gr}=5, a=0.6$, $R=2, M=1.5$, and $\gamma=0.4$ are depicted in Figure 5. It can be seen that velocity increases with increase of permeability parameter or time.

The transient velocity profiles for different values of accelerating parameter $a$ and radiation parameter $R$ at $\mathrm{Gr}$ $=8, k=0.5, t=0.8, M=1$, and $\gamma=0.4$ are plotted in Figure 6. It is observed that velocity decreases with an increase in radiation parameter but decreases with increase 


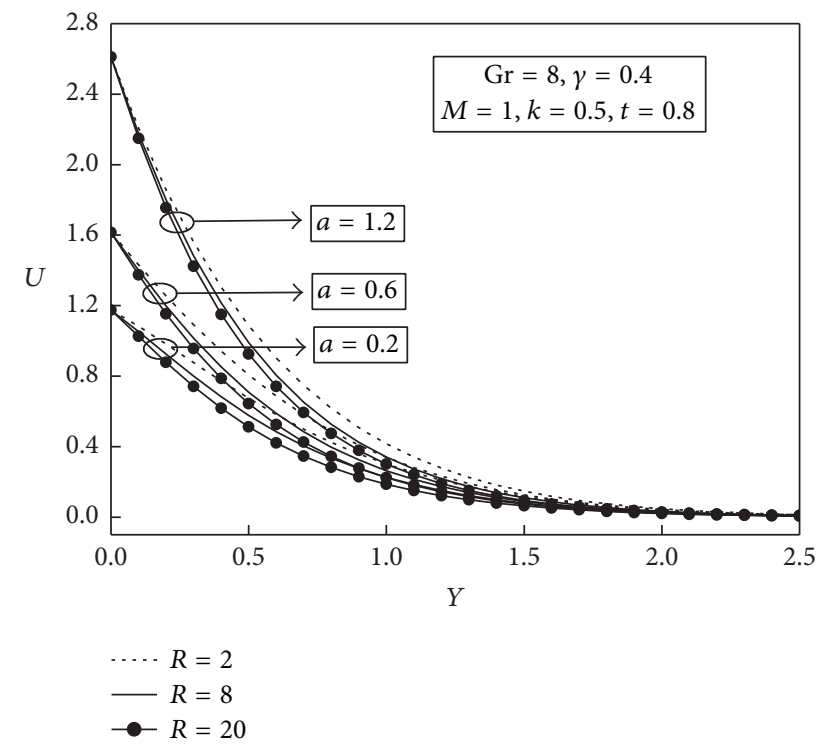

FIGURE 6: Effects of $R$ and $a$ on velocity profiles at $\mathrm{Gr}=8, k=0.5$, $t=0.8, M=1$, and $\gamma=0.4$.

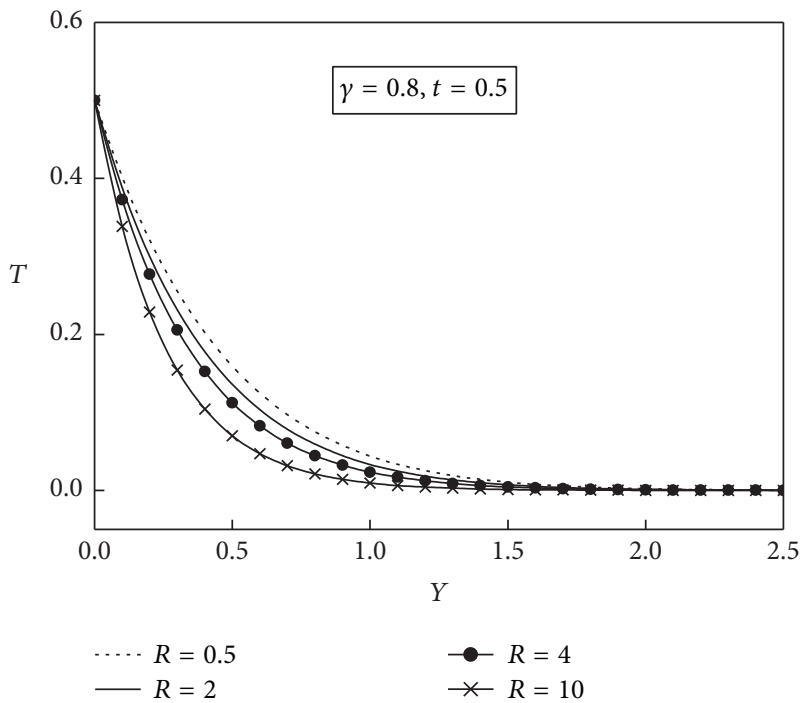

FIGURE 7: Effect of $R$ on temperature profiles at $\gamma=0.8$ and $t=0.5$.

in accelerating parameter. Effects of radiation parameter $R$, suction parameter $\gamma$, and time $t$ on temperature profiles are shown in Figures 7, 8, and 9, respectively. It is observed from these figures that temperature decreases with increased values of radiation parameter or suction parameter but increases with increased values of time.

Effect of Grashof number Gr on skin friction is presented in Figure 10. It is observed from the figure that skin friction decreases with increase of Grashof number. In case of cooling, the plate skin friction decreases continuously with time whereas in case of heating the plate though initially it decreases, but after certain time it tends to increase with increase of time. Effect of accelerating parameter $a$ on skin friction is presented in Figure 11 at $\mathrm{Gr}=-5$ and $5, M=1.2$,



FIGURE 8: Effect of $\gamma$ on temperature profiles at $R=0.2$ and $t=0.5$.

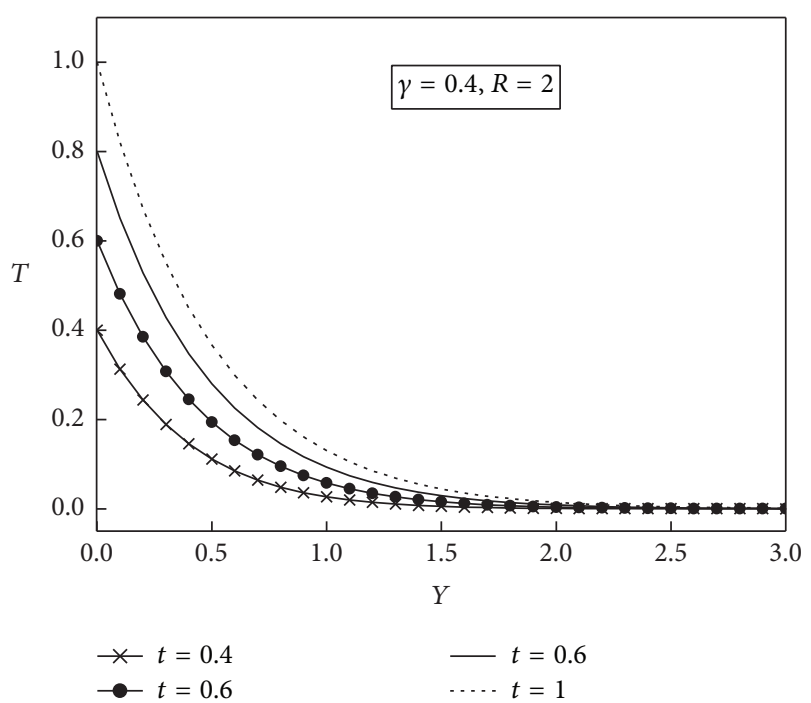

FIGURE 9: Effect of $t$ on temperature profiles at $\gamma=0.4$ and $R=2$.

$\gamma=0.4, R=2$, and $k=0.5$. It is observed that skin friction increases with an increase of accelerating parameter $a$ for both the cases of cooling and heating of the porous plate.

Figure 12 shows the effects of magnetic parameter $M$ and permeability parameter $k$ at $\mathrm{Gr}=5, \gamma=0.4, a=0.3$, and $R=2$ and it is observed that skin friction increases with increase in magnetic parameter but decreases with increase in permeability parameter. Effects of radiation parameter $R$ and suction parameter $\gamma$ on skin friction at $\mathrm{Gr}=5, M=1.2$, $a=0.3$, and $k=0.5$ are depicted in Figure 13 and it can be seen that skin friction increases with increase of radiation parameter or suction parameter.

The Nusselt number for different values radiation parameter $R$ and suction parameter $\gamma$ are shown in Figure 14. The 


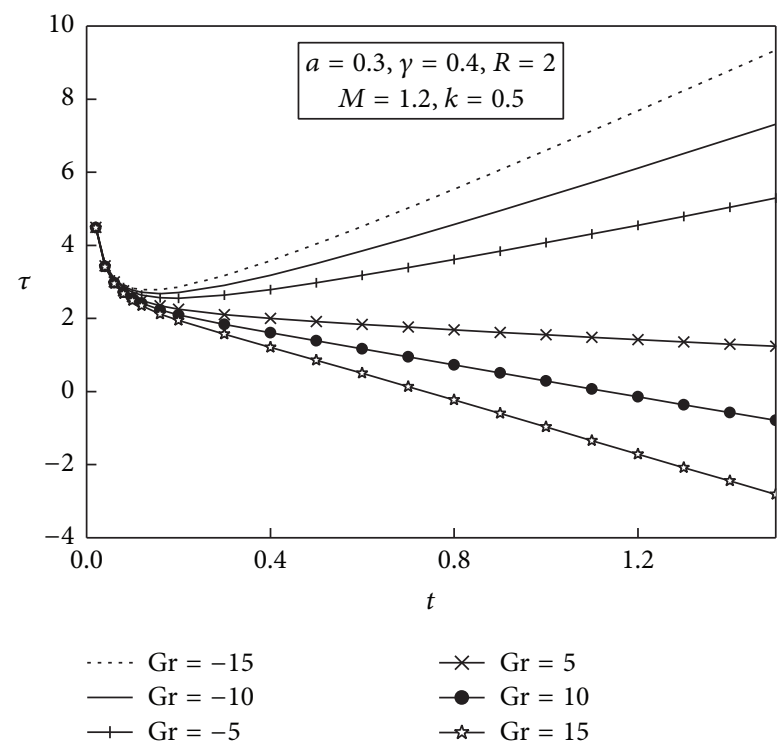

FIGURE 10: Effect of Gr on skin friction at $\gamma=0.4, a=0.3, R=2$, $M=1.2$, and $k=0.5$.

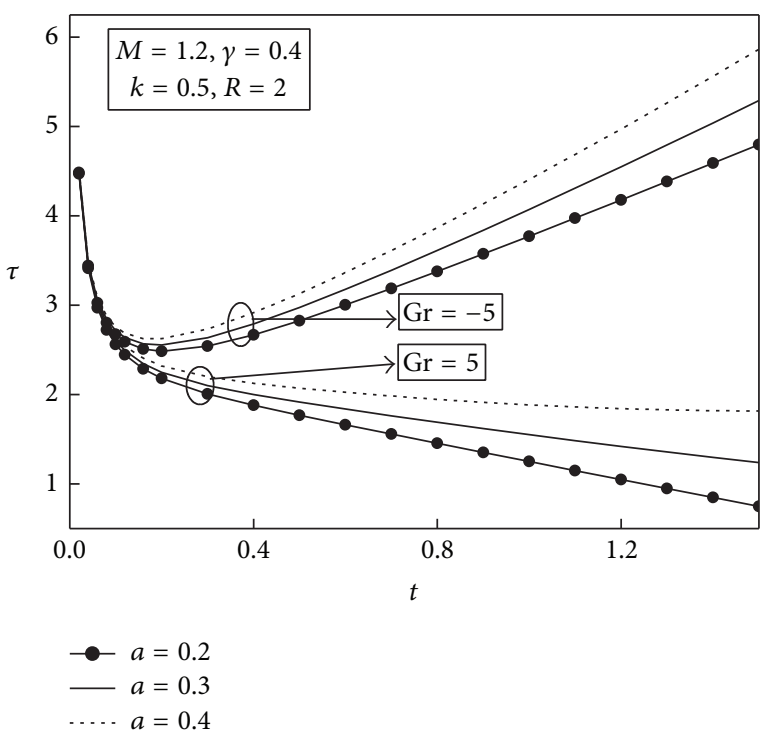

FIGURE 11: Effect of $a$ on skin friction at $M=1.2, \gamma=0.4, R=2$, and $k=0.5$.

rate of heat transfer increases with increase of radiation parameter or suction parameter.

\section{Conclusions}

The analytical study on unsteady one-dimensional natural convective MHD flow of a viscous incompressible and electrically conducting fluid past an exponentially accelerated infinite vertical porous plate through a porous medium with variable temperature is presented. The exact solutions of the dimensionless governing boundary layer equations are

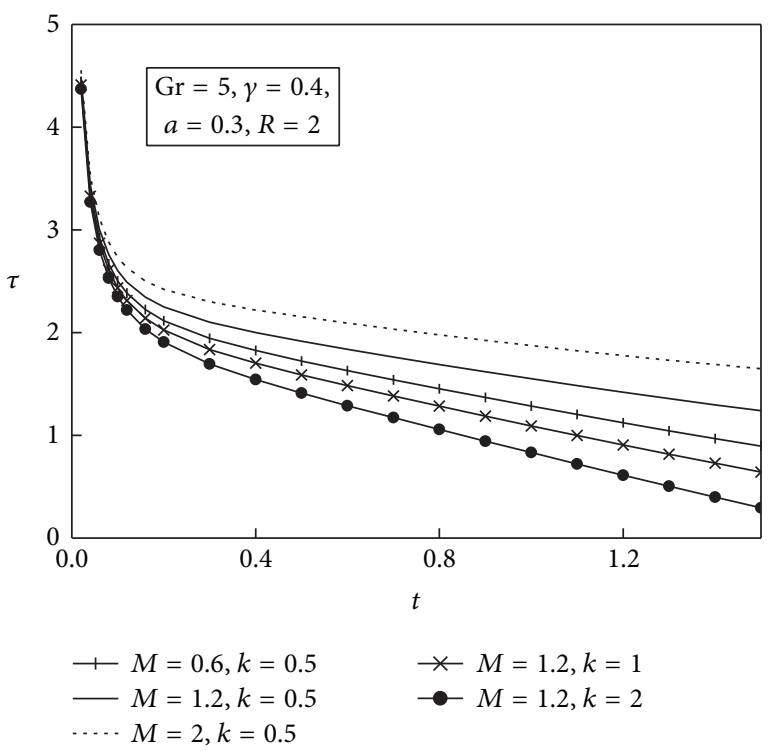

FIgURE 12: Effect of $M$ and $k$ on skin friction at $\mathrm{Gr}=5, \gamma=0.4$, $a=0.3$, and $R=2$.

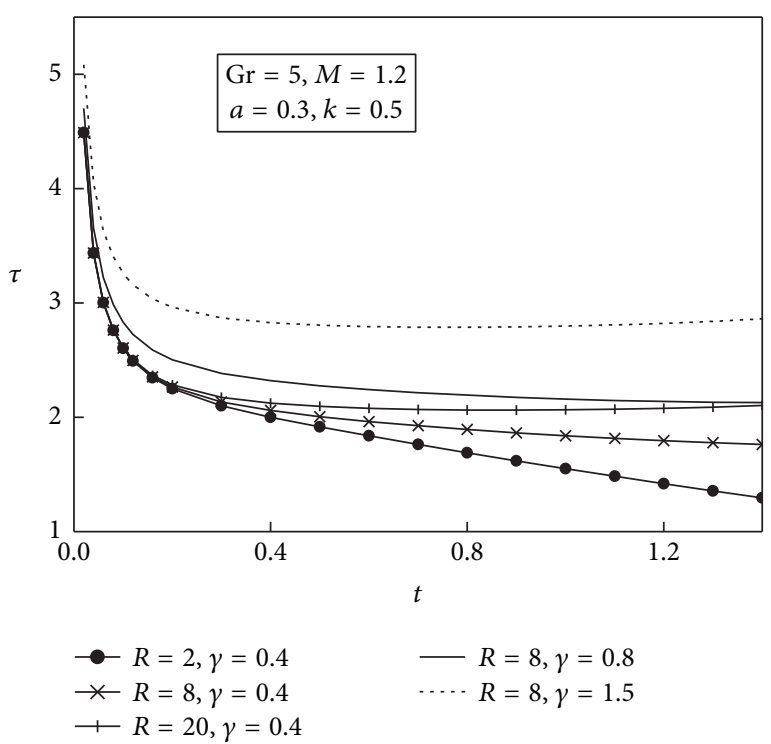

FIgURE 13: Effects of $R$ and $\gamma$ on skin friction at $\mathrm{Gr}=5, M=1.2$, $a=0.3$, and $k=0.5$.

obtained by Laplace transform technique. On the basis of the observations, results, and discussions the conclusions of the present study are as follows:

(1) Velocity increases with increase in Gr or $k$ or $t$ or $a$ but decreases with increase in $M$ or $\gamma$ or $R$.

(2) Temperature increases with increase in $t$ but decreases with increase in $\gamma$ or $R$.

(3) Skin friction increases with increase in $M$ or $\gamma$ or $R$ or $a$ but decreases with increase in Gr or $k$.

(4) Rate of heat transfer increases with increase in $R$ or $\gamma$. 


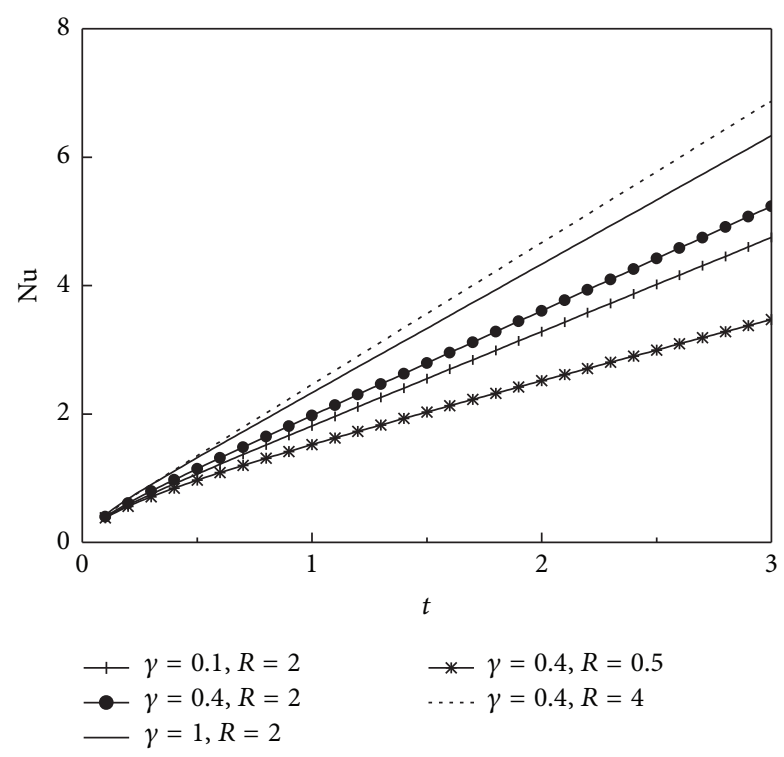

Figure 14: Effect of $\gamma$ and $R$ on Nusselt number.

\section{Nomenclature}

$a^{\prime}$ : Accelerating parameter

$a$ : Dimensionless accelerating parameter

$a^{*}:$ Absorption coefficient

$C_{p}:$ Specific heat at constant pressure

$B_{0}$ : Transverse magnetic field strength

Gr: Grashof number

g: Acceleration due to gravity

$\kappa: \quad$ Thermal conductivity of the fluid

$k^{\prime}$ : Permeability parameter

$k$ : $\quad$ Dimensionless permeability parameter

$M$ : Magnetic field parameter

$\mathrm{Nu}$ : Nusselt number

Pr: Prandtl number

$q_{r}: \quad$ Radiative heat flux in the $y$ direction

$R: \quad$ Radiation parameter

$t^{\prime}$ : Time

$t: \quad$ Dimensionless time

$T^{\prime}$ : Temperature

$T: \quad$ Dimensionless temperature

$T^{\prime}{ }_{w}$ : Temperature of the plate

$T^{\prime}{ }_{\infty}$ : Temperature of the fluid far away from the plate

$u^{\prime}: \quad x$-component of velocity

$u_{0}^{\prime}$ : Velocity of the plate

$U: \quad$ Dimensionless velocity

$v^{\prime}: \quad y$-component of velocity

$y^{\prime}$ : Coordinate axis normal to the plate

$Y:$ Dimensionless coordinate axis normal to the plate.

\section{Greek Symbols}

$\beta$ : Volumetric coefficient of thermal expansion

$\gamma$ : Suction parameter

$v$ : Kinematic viscosity

$\rho$ : Fluid density

$\sigma$ : Electrical conductivity of fluid.

\section{Competing Interests}

The author declares that there is no conflict of interests regarding the publication of this paper.

\section{References}

[1] A. S. Gupta, "Steady and transient free convection of an electrically conducting fluid from a vertical plate in the presence of a magnetic field," Applied Scientific Research, vol. 9, no. 1, pp. 319-333, 1960.

[2] A. K. Singh and N. Kumar, "Free-convection flow past an exponentially accelerated vertical plate," Astrophysics and Space Science, vol. 98, no. 2, pp. 245-248, 1984.

[3] B. K. Jha, R. Prasad, and S. Rai, "Mass transfer effects on the flow past an exponentially accelerated vertical plate with constant heat flux," Astrophysics and Space Science, vol. 181, no. 1, pp. 125134, 1991.

[4] R. Muthucumaraswamy, K. Sathappan, and R. Natarajan, "Heat transfer effects on flow past an exponentially accelerated vertical plate with variable temperature," Theoretical and Applied Mechanics, vol. 35, no. 4, pp. 323-331, 2008.

[5] R. Muthucumaraswamy, K. E. Sathappan, and R. Natarajan, "Heat and mass transfer effects on exponentially accelerated vertical plate with uniform magnetic field," Journal of Engineering, Annals, vol. 6, no. 3, pp. 188-193, 2008.

[6] V. Rajesh, S. Varma, and V. Kumar, "Radiation and mass transfer effects on MHD free convection flow past an exponentially accelerated vertical plate with variable temperature," ARPN Journal of Engineering and Applied Sciences, vol. 4, no. 6, pp. 20 26, 2009.

[7] V. Rajesh and A. J. Chamkha, "Unsteady convective flow past an exponentially accelerated infinite vertical porous plate with Newtonian heating and viscous dissipation," International Journal of Numerical Methods for Heat \& Fluid Flow, vol. 24, no. 5, pp. 1109-1123, 2014.

[8] R. Srinivasa, G. Aruna, S. Naidu, S. Varma, and M. Rashidi, "Chemically reacting fluid flow induced by an exponentially accelerated infinite vertical plate in a magnetic field and variable temperature via LTT and FEM," Theoretical and Applied Mechanics, vol. 43, no. 1, pp. 49-83, 2016.

[9] A. S. Gupta, I. Popp, and V. M. Soundalgekar, "Free convection effects on the flow past an accelerated vertical plate in an incompressible dissipative fluid," Revue Roumaine des Sciences Techniques. Serie de Mecanique Appliquee, vol. 24, no. 4, pp. 561$568,1979$.

[10] N. G. Kafousias and A. A. Raptis, "Mass transfer and free convection effects on the flow past an accelerated vertical infinite plate with variable suction or injection," Revue Roumaine des Sciences Techniques-Serie de Mecanique Appliquee, vol. 26, pp. $11-22,1981$.

[11] C. Ping and I. Pop, “Transient free convection about a vertical flat plate embedded in a porous medium," International Journal of Engineering Science, vol. 22, no. 3, pp. 253-264, 1984.

[12] B. K. Jha, "MHD free-convection and mass-transform flow through a porous medium," Astrophysics and Space Science, vol. 175, no. 2, pp. 283-289, 1991.

[13] E. Magyari, I. Pop, and B. Keller, "Analytical solutions for unsteady free convection in porous media," Journal of Engineering Mathematics, vol. 48, no. 2, pp. 93-104, 2004.

[14] R. C. Chaudhary and A. Jain, "Combined heat and mass transfer effects MHD free convection flow past an oscillating plate 
embedded in porous medium," Romanian Journal of Physics, vol. 52, pp. 505-524, 2007.

[15] R. C. Chaudhary and A. Jain, "Magnetohydrodynamic transient convection flow past a vertical surface embedded in a porous medium with oscillating temperature," Turkish Journal of Engineering and Environmental Sciences, vol. 32, no. 1, pp. 13-22, 2008.

[16] R. C. Chaudhary and A. Jain, "MHD heat and mass diffusion flow by natural convection past a surface embedded in a porous medium," Theoretical and Applied Mechanics, vol. 36, no. 1, pp. $1-27,2009$.

[17] R. C. Chaudhary, A. Jain, and M. C. Goyal, "Free convection effects on MHD flow past an infinite vertical accelerated plate embedded in porous media with constant heat flux," Matemáticas Enseñanza Universitaria, vol. 17, no. 2, pp. 73-82, 2009.

[18] V. Rajesh, "MHD free convection flow past an accelerated vertical porous plate with variable temperature through a porous medium," Acta Technica Corviniensis-Bulletin of Engineering, vol. 2, pp. 91-96, 2010.

[19] V. Rajesh, M. Mallesh, and O. A. Bég, “Transient MHD free convection flow and heat transfer of nanofluid past an impulsively started vertical porous plate in the presence of viscous dissipation," Procedia Materials Science, vol. 10, pp. 80-89, 2015.

[20] S. M. Ibrahim, "Effects of chemical reaction on dissipative radiative MHD flow through a porous medium over a nonisothermal stretching sheet," Journal of Industrial Mathematics, vol. 2014, Article ID 243148, 10 pages, 2014. 


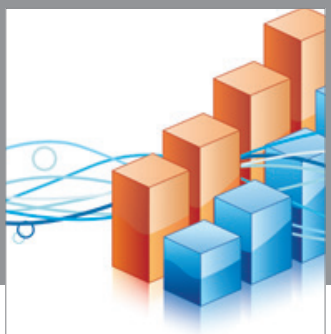

Advances in

Operations Research

vatem alat4

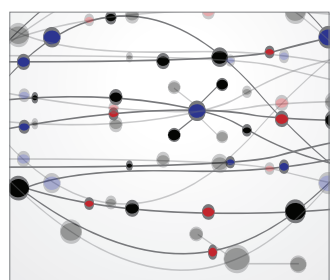

\section{The Scientific} World Journal
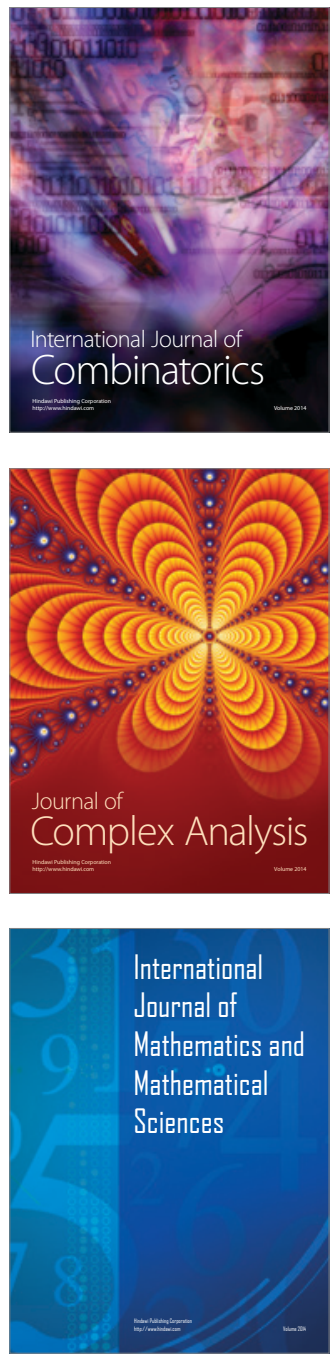
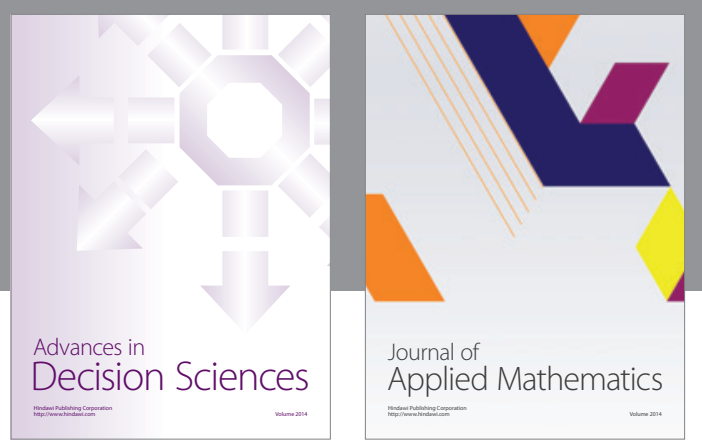

Algebra

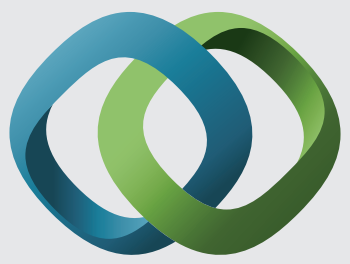

\section{Hindawi}

Submit your manuscripts at

https://www.hindawi.com
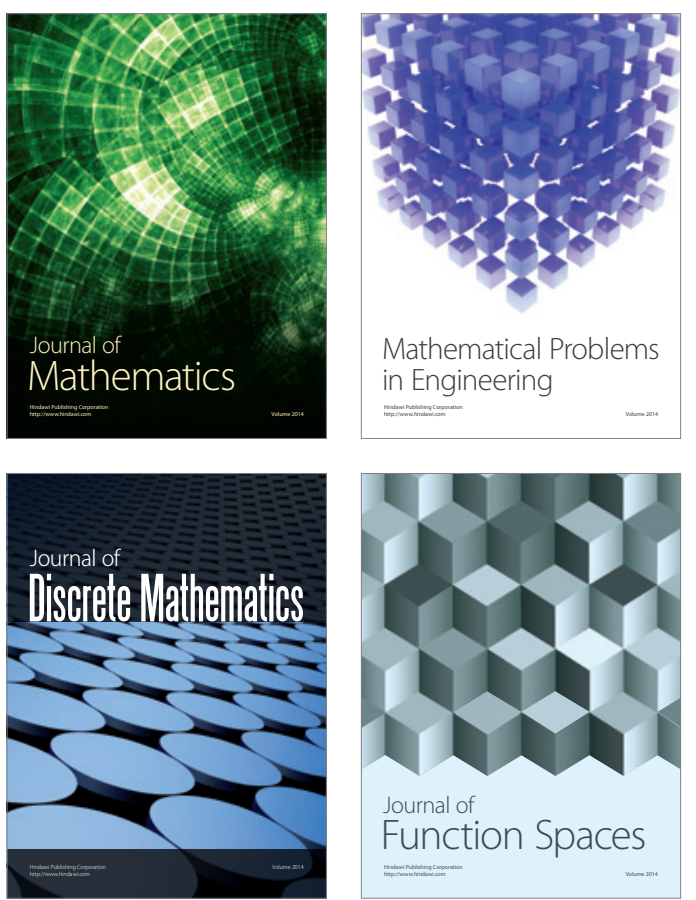

Mathematical Problems in Engineering
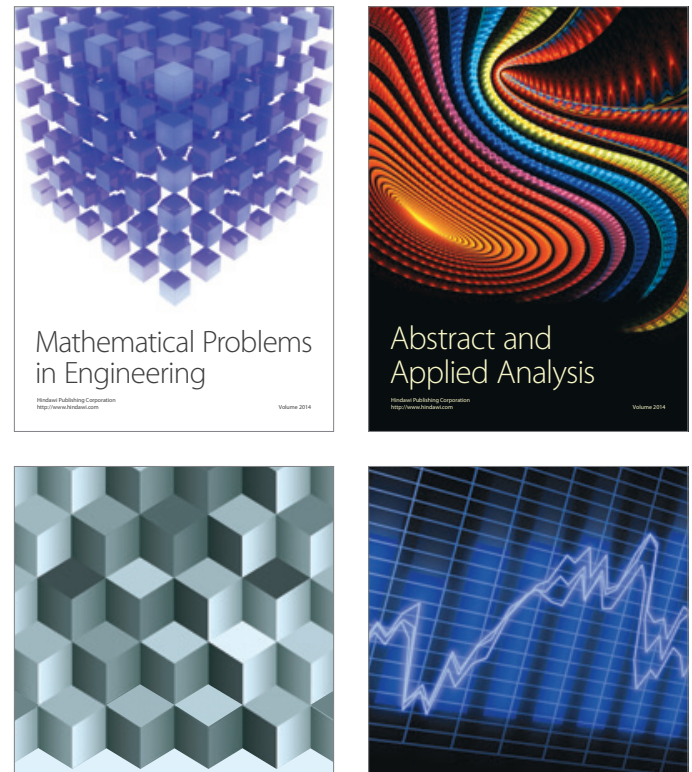

Journal of

Function Spaces

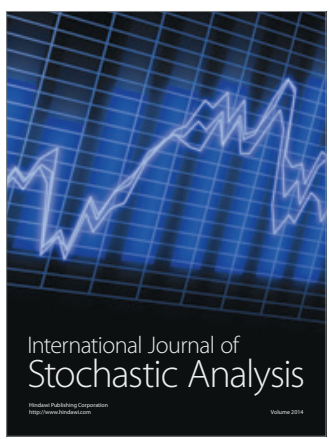

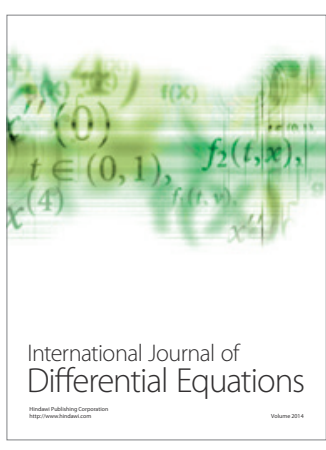
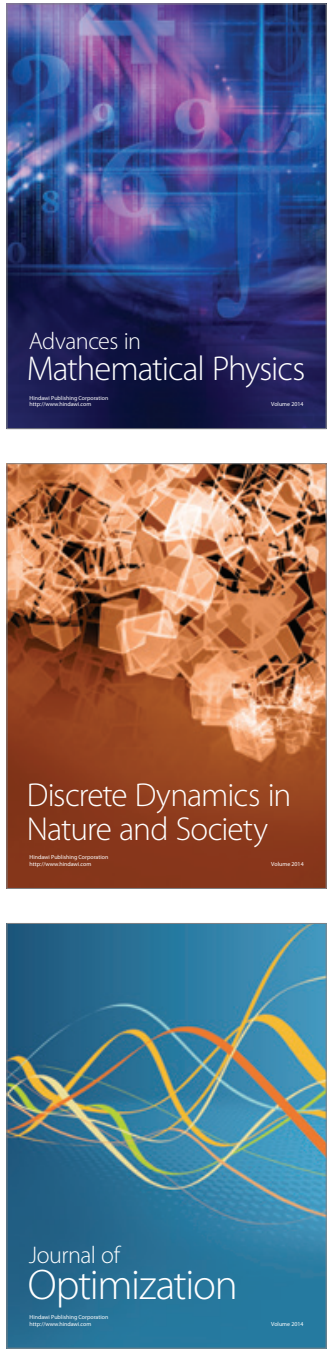